\title{
Reprise en sous-œuvre du château des Chevaliers Teutoniques de Malbork
}

\section{E. DEMBICKI \\ W. ODROBINSKI}

Université technique de Gdansk, Pologne

G. SANGLERAT

Expert agréé par la Cour de cassation. Lyon

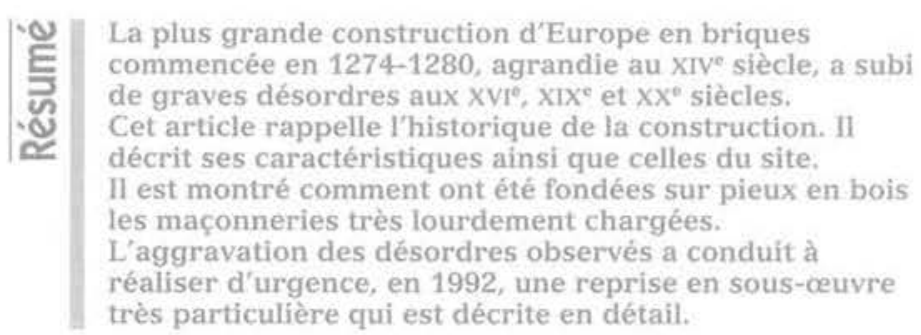

\section{Underpining of Malbork Castel}




\section{Aperçu historique}

Aux $\mathrm{XI}^{e}$ et $\mathrm{XI} \mathrm{II}^{e}$ siècles, la Pologne est morcelée en duchés de plus en plus nombreux, divisés par la mésentente et menacés par les invasions tartares ou par l'expansion allemande (émigration pacifique de bourgeois et paysans, ou guerres ( féodales »).

Vers l'an 1000, le pays compris entre les estuaires de la Vistule et du Niemen est habité par une population baite, parente des Lettons et des Lithuaniens, les Bruzi (ou vieux Prussiens). Pailens pratiquant encore des sacrifices humains, ils accueillent mal les tentatives d'évangélisation menées par l'Empire, et reprises par les rois de Pologne.

Au début du Xmre siècle, se produit une vive réaction païenne de ces populations. Incapable d'y résister, le duc polonais Conrad de Mazovie fait intervenir, sans grand succès, divers ordres de chevalerie d'Europe occidentale, puis fait appel, en 1226, aux Chevaliers Teutoniques.

L'Ordre des Chevaliers Teutoniques est un ordre religieux, fondé par les bourgeois de Brême et de Lübeck pendant le siège d'Acre (1191), d'abord hospitalier avant d'être transformé en ordre militaire, soumis à la règle des Templiers. En principe ouvert à toutes nationalités, il ne recrute en pratique que des Allemands, et il acquiert au xille siècle des possessions considérables en Terre Sainte et de nombreuses donations en Europe méditerranéenne, et surtout en Allemagne. En 1237, 1'Ordre Teutonique incorpore les Chevaliers Porte-Glaive, ordre militaire fondé en 1202, basé à Riga, qui se rend maître de la majeure partie de la Lettonie et de l'Estonie avant de voir son expansion vers l'est stoppée en 1242 sur les bords du lac Peïpous.

Après son expulsion de Hongrie, l'Ordre des Chevaliers Teutoniques connait un rapide essor pendant la guerre particulièrement acharnée menée contre la Prusse paienne (1230-1283).

L'Ordre acquiert les mêmes droits que les princes d'Empire, il favorise une colonisation allemande active qui remplace, dans des centaines de villages protégés par des châteaux, les vieux Prussiens exterminés ou refoulés.

Sur les terres concédées en 1226 par le duc de Mazovie, les Chevaliers Teutoniques construisirent un premier château à Thorn (Torum) en 1250. Ensuite, ils érigèrent les châteaux de Kulm (Chelmno) et de Marienwerder (Kwidzyn).

Le château des Chevaliers Teutoniques de Malbork fait partie des plus importants monuments de l'architecture gothique de défense en Europe.

Édifié sur la rive droite du Nogat, le bras oriental de la basse Vistule, à une vingtaine de kilomètres en amont d'Elbing, il est considéré comme le plus grand édifice de ce genre, construit entièrement en briques. Ce n'est pas seulement un superbe monument historique, mais aussi un exemple typique de l'art de construire médiéval.

Sa construction fut commencée vers 1274 par l'Ordre des Chevaliers Teutoniques à l'initiative du Maître provincial Conrad de Tiberg. Vers 1280 fut construit le Château Haut (Hochburg), véritable donjon en forme de carré avec une cour en arcades, qui comportait, entre autres, un réfectoire, un capitulaire et une chapelle consacrée à la Sainte Vierge, ornée d'un très beau portail dont la Porte dorée. La forteresse reçut le nom de Marienburg en I'honneur de la Vierge Marie sous le patronage de laquelle était placé l'Ordre depuis ses origines hiérosolymitaines.

Jusqu'à la fin du XiI" siècle, le château fut entouré d'une enceinte qui cernait toute la colline et servait en même temps de muraille de soutènement. A cette époque furent construites aussi deux tours; celle de l'extérieur contenait les installations sanitaires.

C'est dans le Hochburg que résida à partir de 1309, le Grand Maitre de l'Ordre.

Durant le XIV siècle l'ensemble fut agrandi. L'extension du Château Haut (cf. A, Fig. 1) se fï du côté de l'aile Est, le capitulaire et la chapelle furent refaits. Les dépendances furent transformées en un superbe Château Moyen (Mittelburg), muni d'un grand réfectoire (salle royale en 1320). Du côté de l'aile Est, en 1398 fut élevé le palais du Grand Maître (cf. B, Fig. 1). On construisit également du côté nord, dans le prolongement du Mittelburg une annexe, le Vorburg, occupé par les écuries, les étables, les magasins de vivres, les divers ateliers et l'arsenal. Un pont reliait le Hochburg à la rive gauche du Nogat. Les deux châteaux furent entourés de murailles et équipés d'immenses fortifications qui rejoignirent les remparts de la ville.

L'ensemble des bâtiments fut achevé au milieu du XIV ${ }^{e}$ siècle. Entourée d'une double enceinte garnie de tours et précédée d'un fossé profond, la forteresse de Marienburg couvrait une superficie d'une douzaine
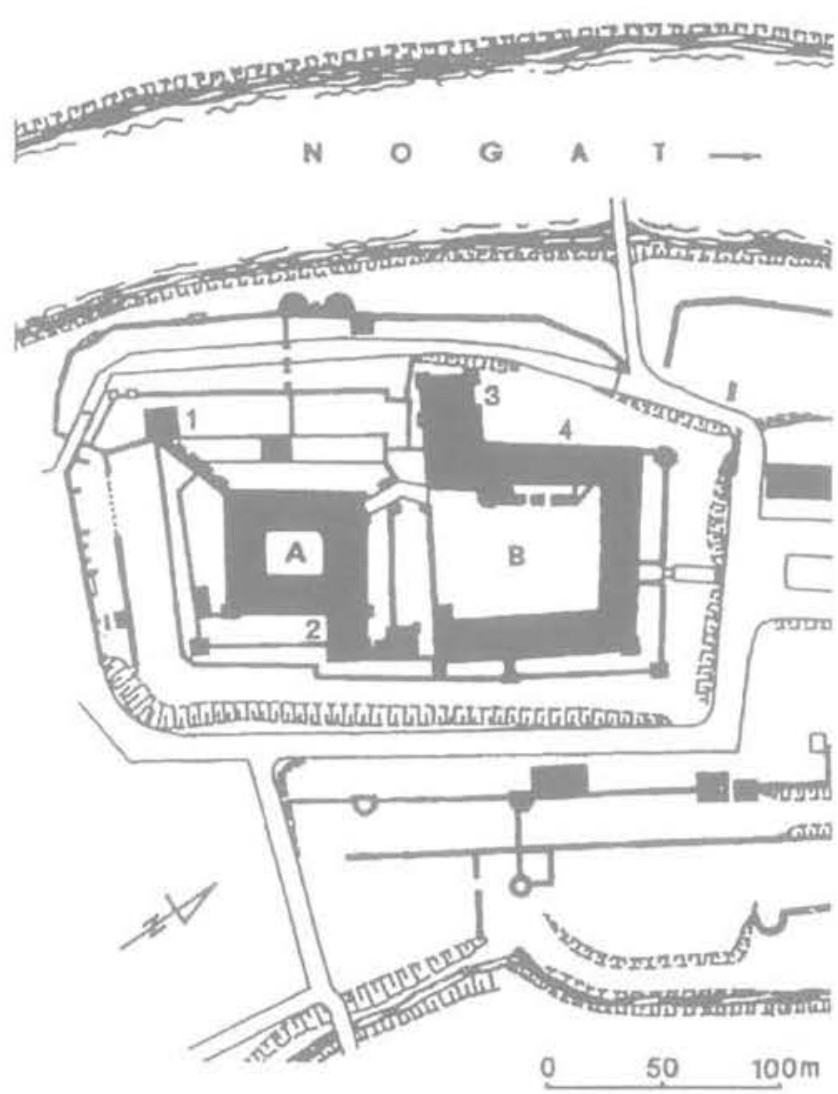

FG:-1/ Plan du château. Plan of Castle.

A. Château Haut, High Castle,

B. Château Moyen, Middle Castle.

1. Tour sanitaire, Sanitary tower.

2. Église de la Sainte-Vierge et chapelle de Sainte-Anne, Holy Virgin Church and Saint Anne Chapel.

3. Palais du Grand Maitre, Palace of the Great Master.

4. Grand Réfectoire, Mess Hall. 
d'hectares. Construite en briques, selon la tradition de l'Allemagne du Nord, la forteresse constitue l'une des plus remarquables réalisations de l'art militaire des Teutoniques. Dès la fin du XIII siècle, une ville se développa du côté du Hochburg, couvrant une superficie de six hectares, elle aussi ceinte de murailles. A son apogée au XIV siècle, sa population ne dépassa guère les deux mille âmes. Mais la présence du Grand Maître et de ses Grands Officiers, dont le Maître provincial de Prusse, allait faire de Marienburg (Malbork) le symbole de la présence allemande en terre prussienne.

Malgré les guerres, les incendies et la perte de l'importance militaire, jusqu'aux années $70 \mathrm{du}$ XVIII siècle aucun travail important ne fut entrepris, et le château se trouvait dans un bon état. C'est seulement à ce moment que de grandes détériorations apparurent à l'intérieur; le château fut transformé en casernes, magasins, ateliers et logements pour les ouvriers.

Les travaux de rénovation à grande échelle furent entrepris en 1804 pour être ensuite interrompus par les guerres de Napoléon. Ils furent repris en 1817 et poursuivis jusqu'à la moitié du XIXe siècle. Cette période appelée «la reconstruction romantique du XIX siècle» fut d'ailleurs très critiquée. Tous les éléments d'architecture postérieurs au Moyen Age furent éliminés tant dans la structure du bâtiment qu'à l'intérieur. On tâcha de redonner à chaque partie du bâtiment son aspect d'origine, grâce à des éléments d'architecture d'époque extraits des décombres.

En 1945 le château, transformé par l'armée allemande en forteresse, se défendit durant deux mois contre les attaques de l'armée soviétique. Ce complexe monumental fut alors détruit à $50 \%$ environ. Sa reconstruction, conforme aux exigences de la technique moderne dura plus d'une dizaine d'années, et les travaux ne furent pratiquement jamais interrompus.

\section{Dégradation du mur Ouest du château moyen}

Les problèmes liés à la détérioration du mur Ouest et des voûtes du Grand Réfectoire (Fig. 3 et 4) ont une longue histoire. Le mur Ouest se trouve à environ $80 \mathrm{~m}$ de la rive actuelle d'un bras de l'estuaire de la Vistule appelé fleuve de Nogat (Fig. 2); ce bras est le plus long du delta de la Vistule. Dans le passé, il y avait à cet endroit un fossé. Le terrain descendait naturellement vers le Nogat, donc les murs longitudinaux de cette partie du château, parallèles au fleuve, ont eu différents systemes de fondation.

Le Grand Réfectoire, situé dans l'aile attenante au palais du Grand Maitre; est une des rares salles gothiques à destination laique qui existent encore en Europe, et constitue l'une des plus précieuses parties du château. Elle mesure $30,35 \mathrm{~m} \times 15,30 \mathrm{~m}$ sur $9 \mathrm{~m}$ de haut. Sa superbe voûte en feuilles de palmes (en éventail) s'appuie sur trois piliers de granite (Fig. 3). Au-dessous de la salle, il y a deux étages de caves et à côté une cuisine. Au-dessus un toit à double pente abrite un grenier qui servait à différents usages (Fig. 5 et 10).

Des traces de désordres, dont la reprise a été engagée dès l'origine de la construction, sont apparues au moment de la mise à découvert des murs devant être à nouveau réparés. Les premiers signes révélateurs de fissurations sur les murs transversaux et sur les voûtes datent du $\mathrm{XVI}^{\mathrm{e}}$ siècle. Depuis, ces fissures réapparaissaient de temps en temps, et chaque fois on les bouchait avec du mortier.

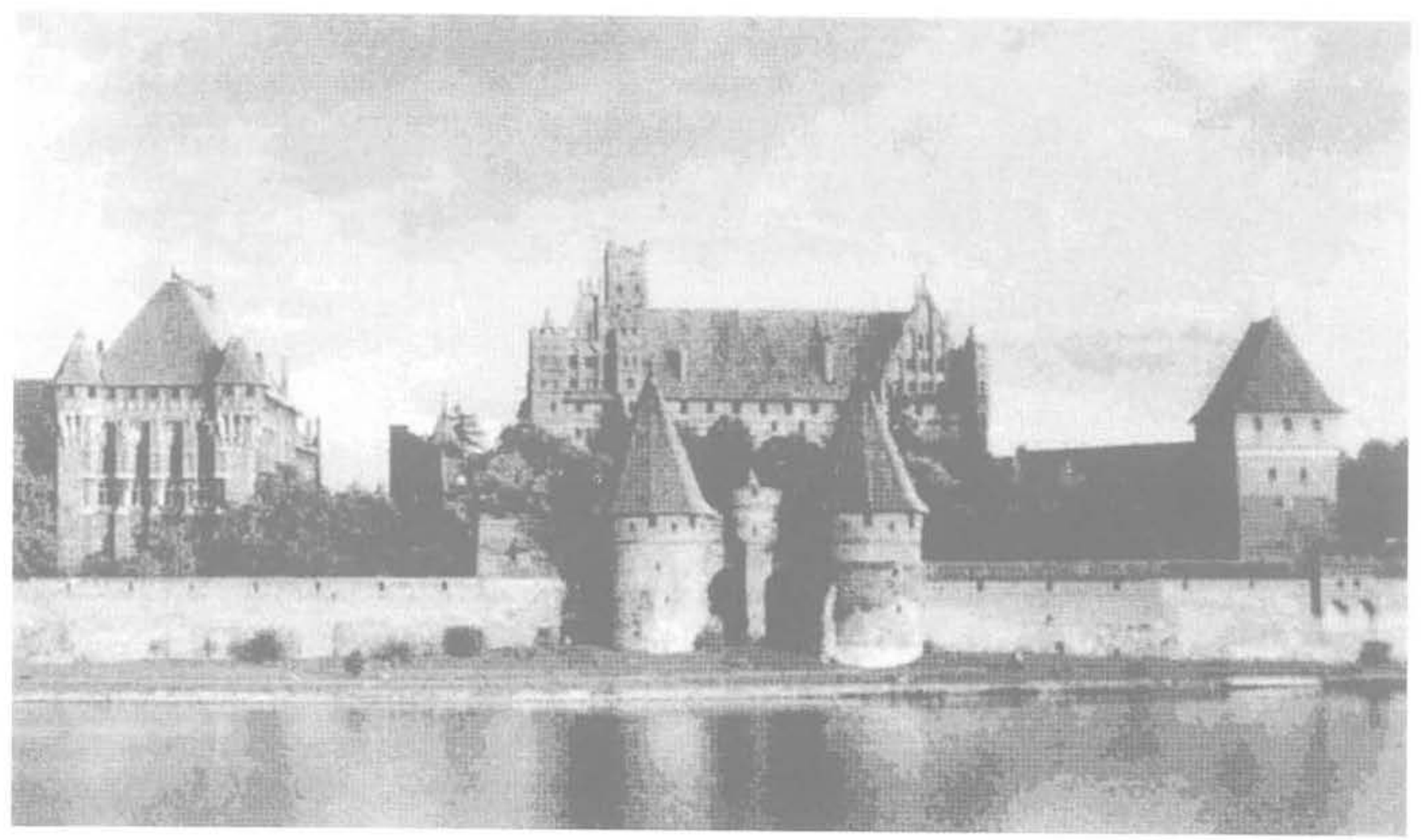

FG.2 Vue du château du côté du fleuve (photo A. Pacek).

Castle seen from the river. 


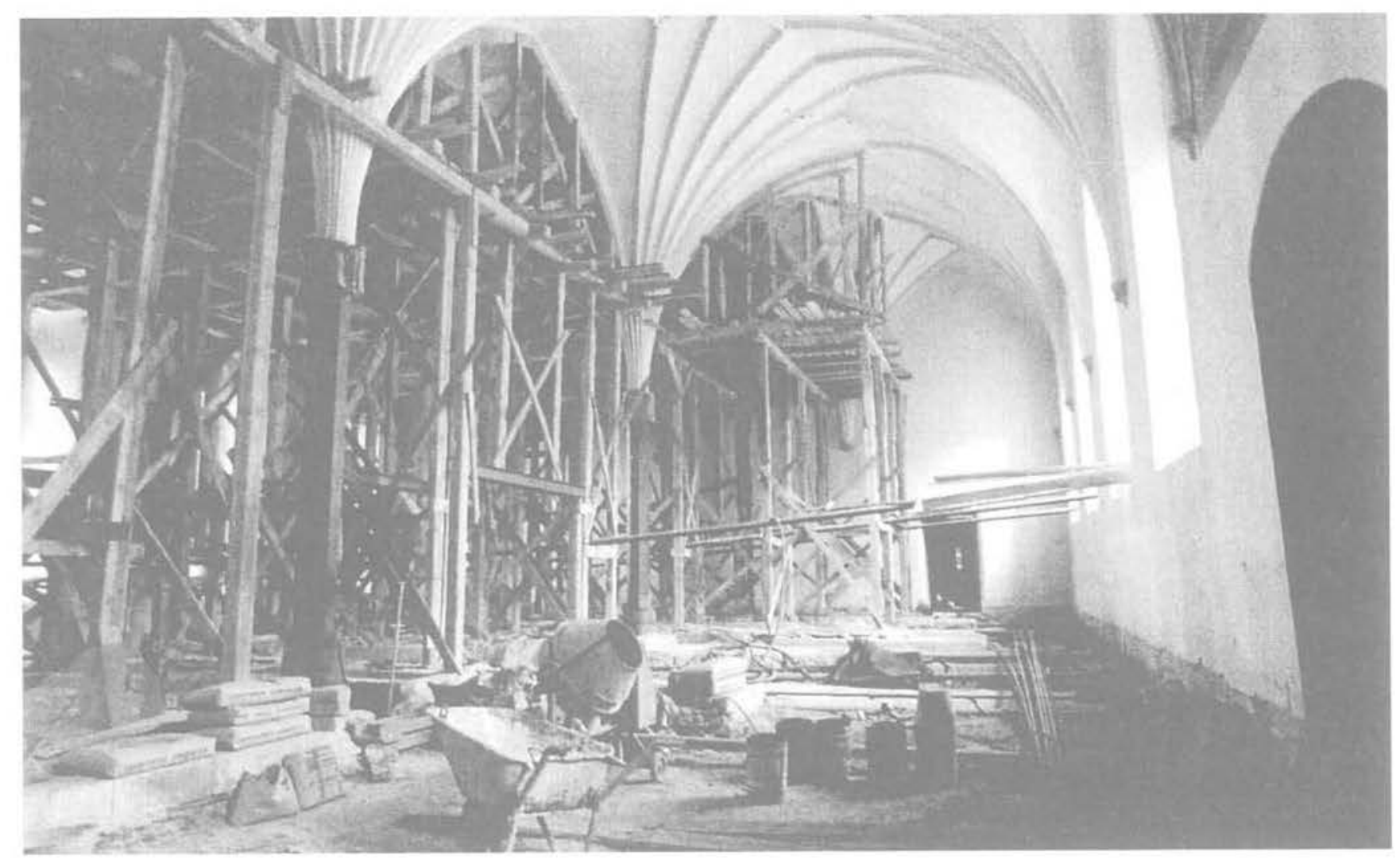

FIG.3 Intérieur du Grand Réfectoire (photo A. Pacek). Inside the Great Mess Hall.

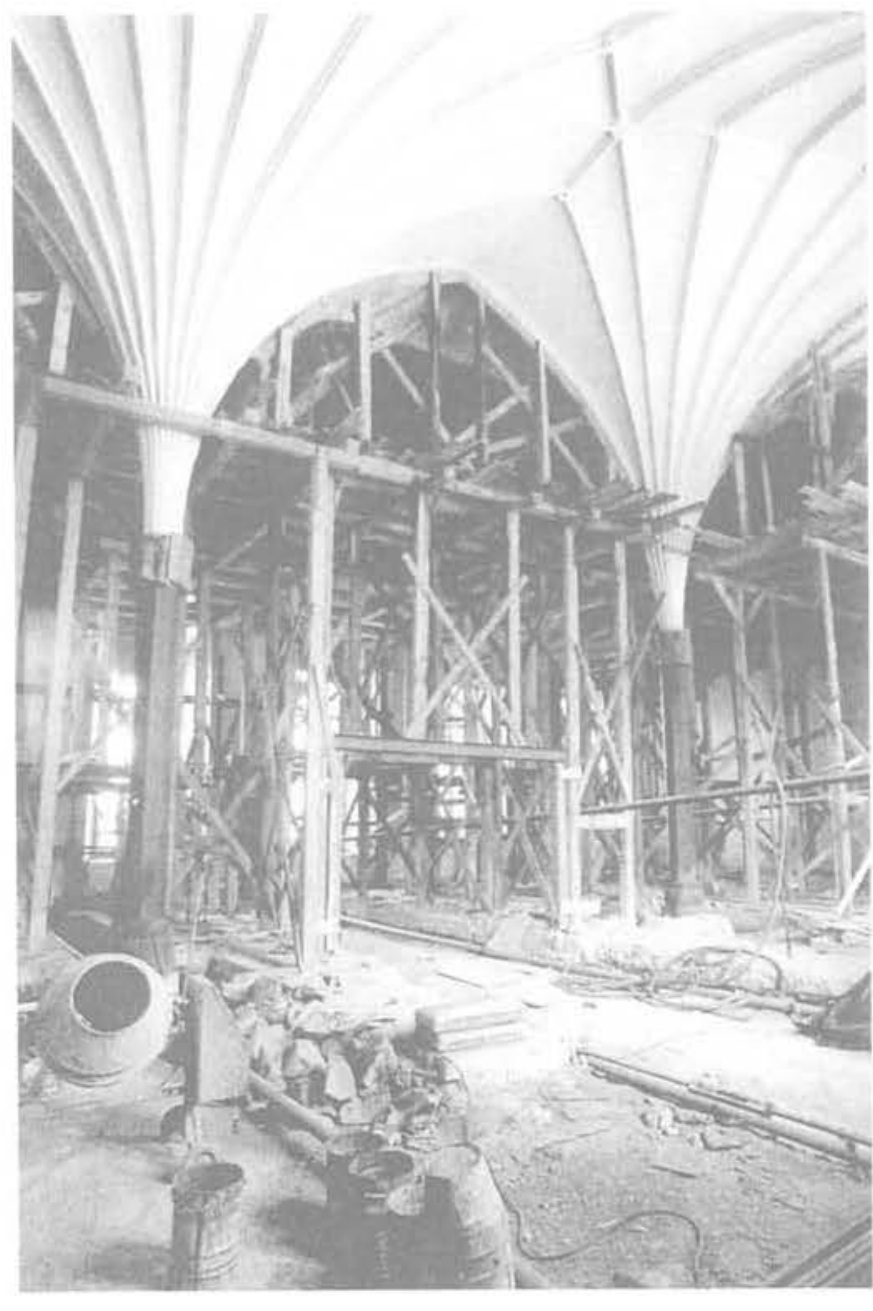




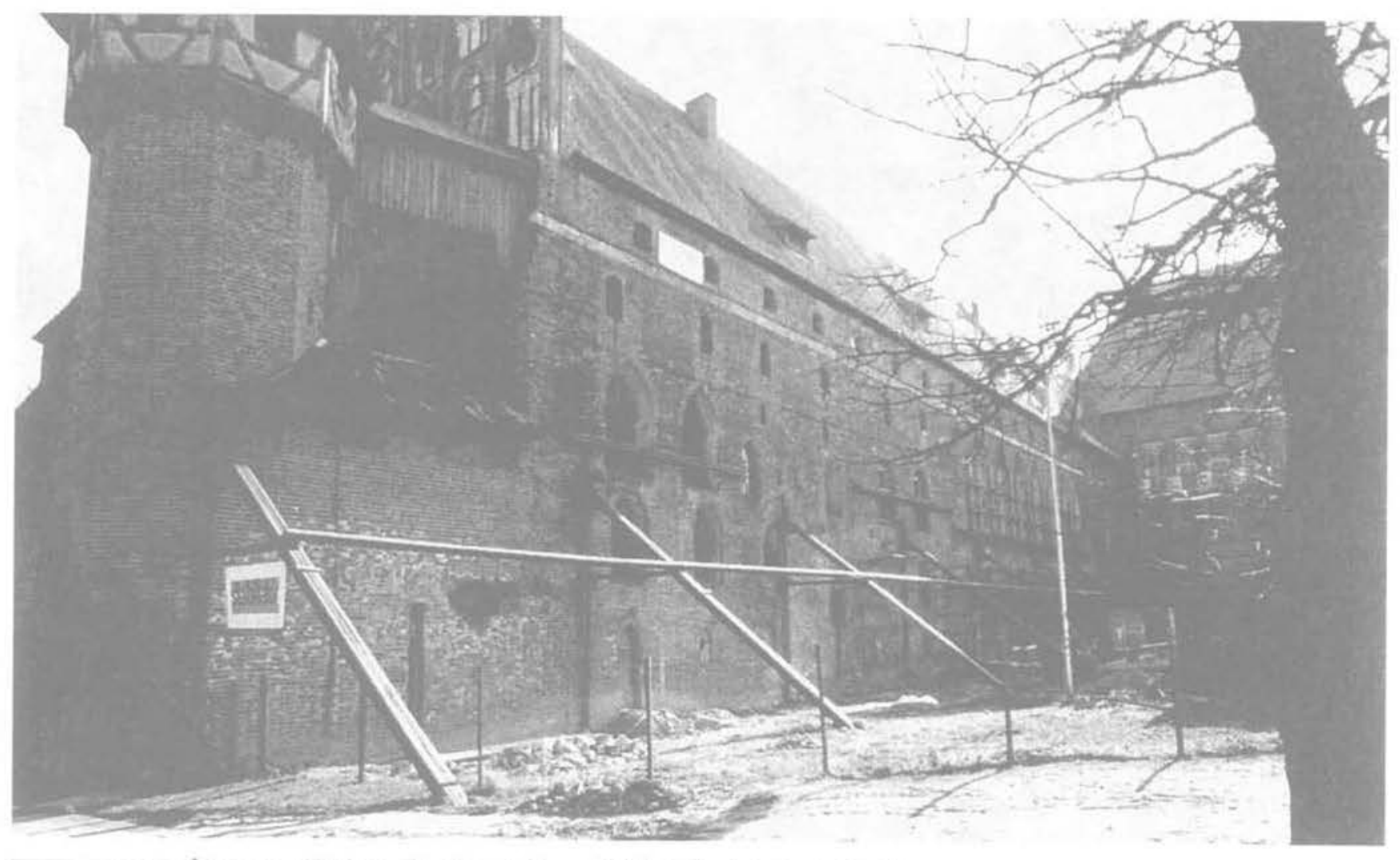

FG.5 Étayage extérieur, aile Ouest, Grand Réfectoire (photo A. Pacek). Battered props, west wing, Great Mess Hall,

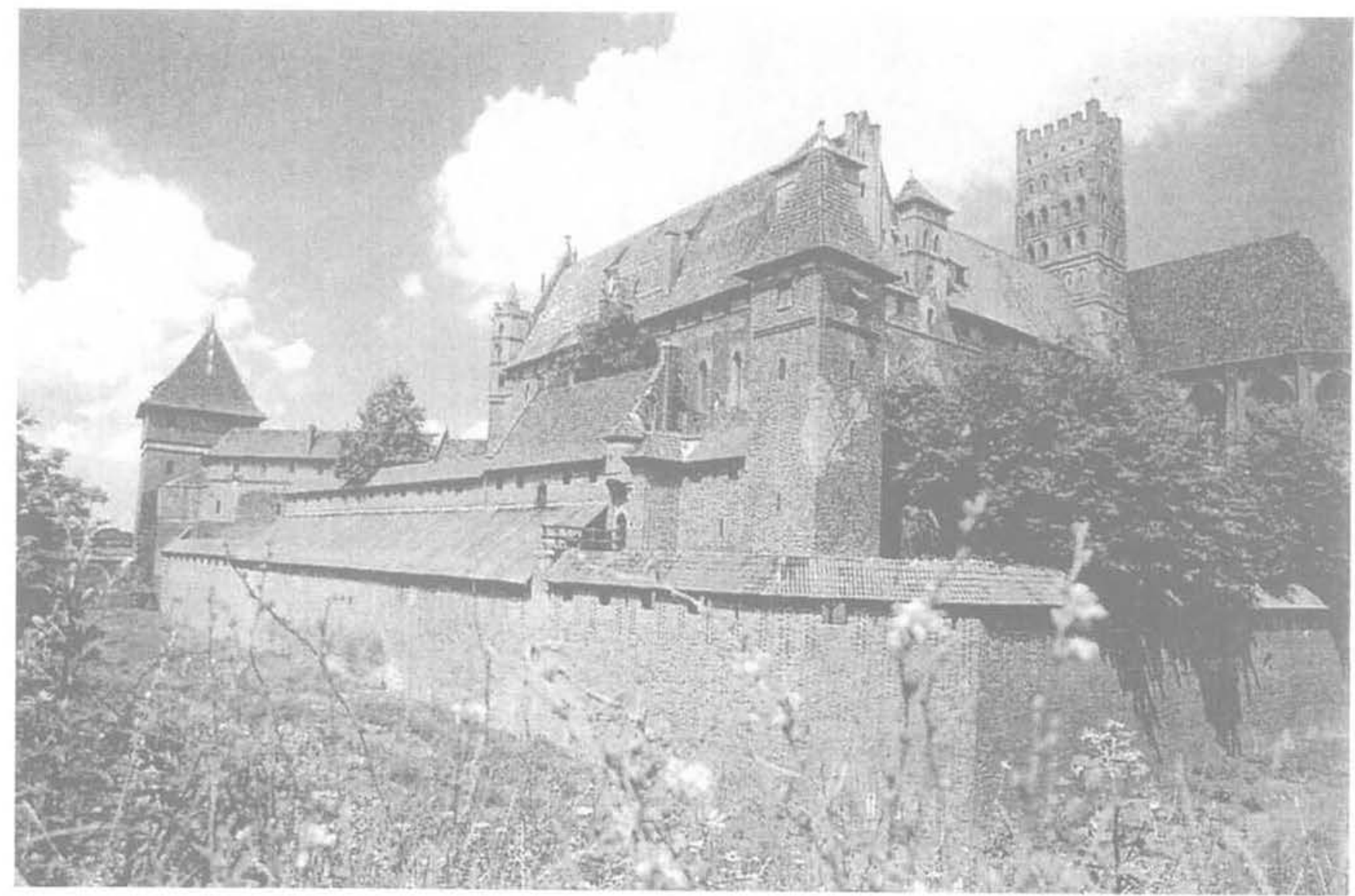

FIG.6 Côté sud-est du Château Haut (photo R. Czewinski et A. Stachurski).

South-East side of the High Castle. 


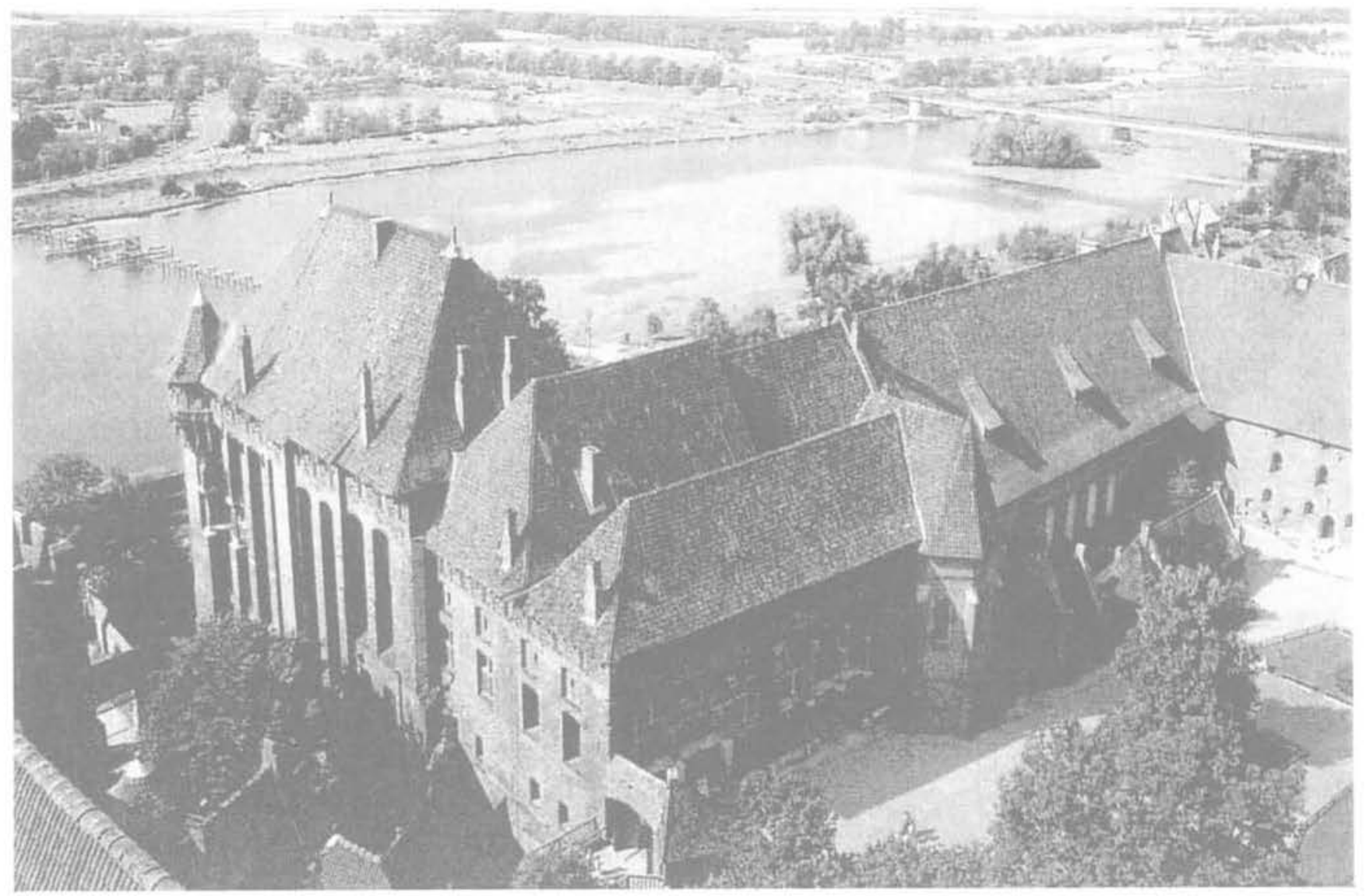

FIG.7 Vue de la tour sur le palais du Grand Maitre (photo R. Czewinski et A. Stachurski). View of the Palace of Grand Master from the Castle tower.

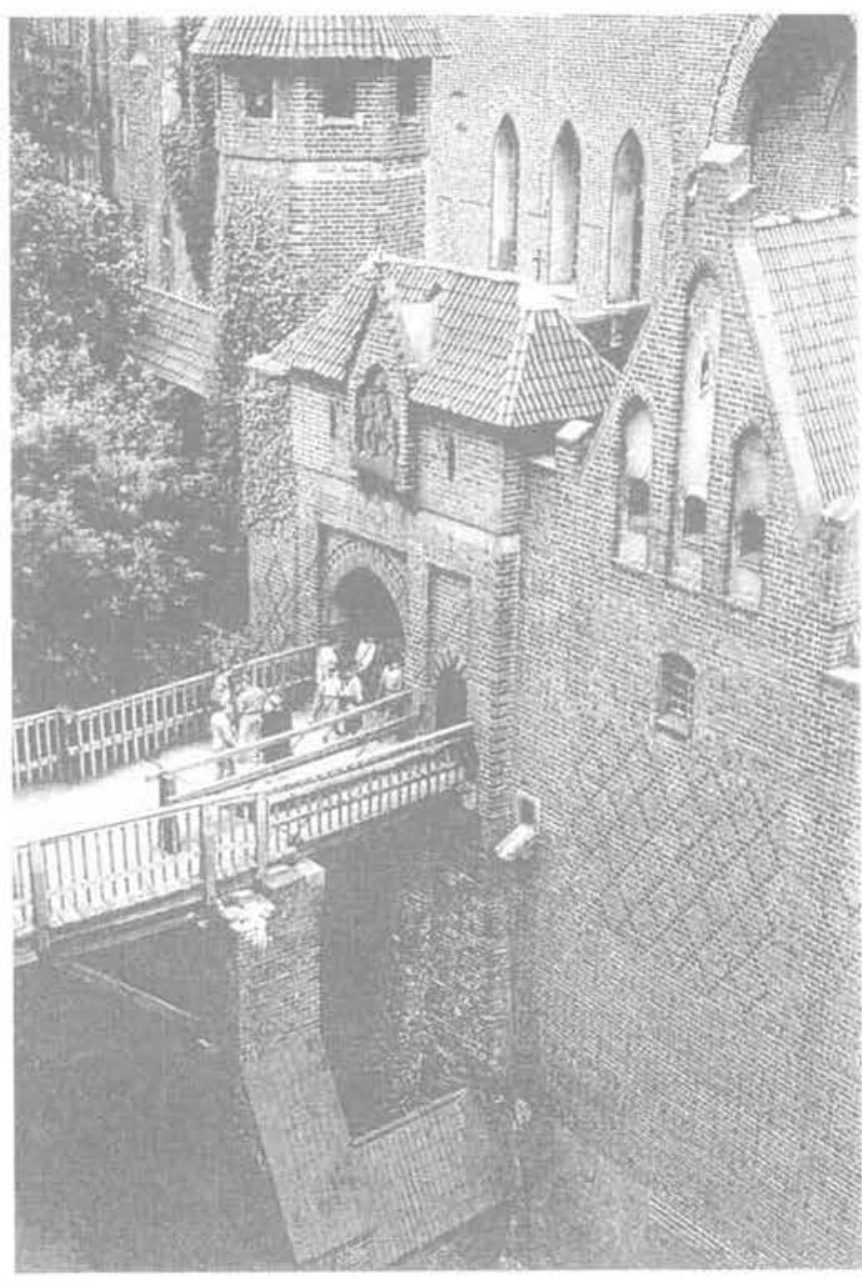

F16:8 Le pont-levis et la porte du Château Haut (photo R. Czewinski et A. Stachurski). The drawbridge and the gate of the High Castle. 


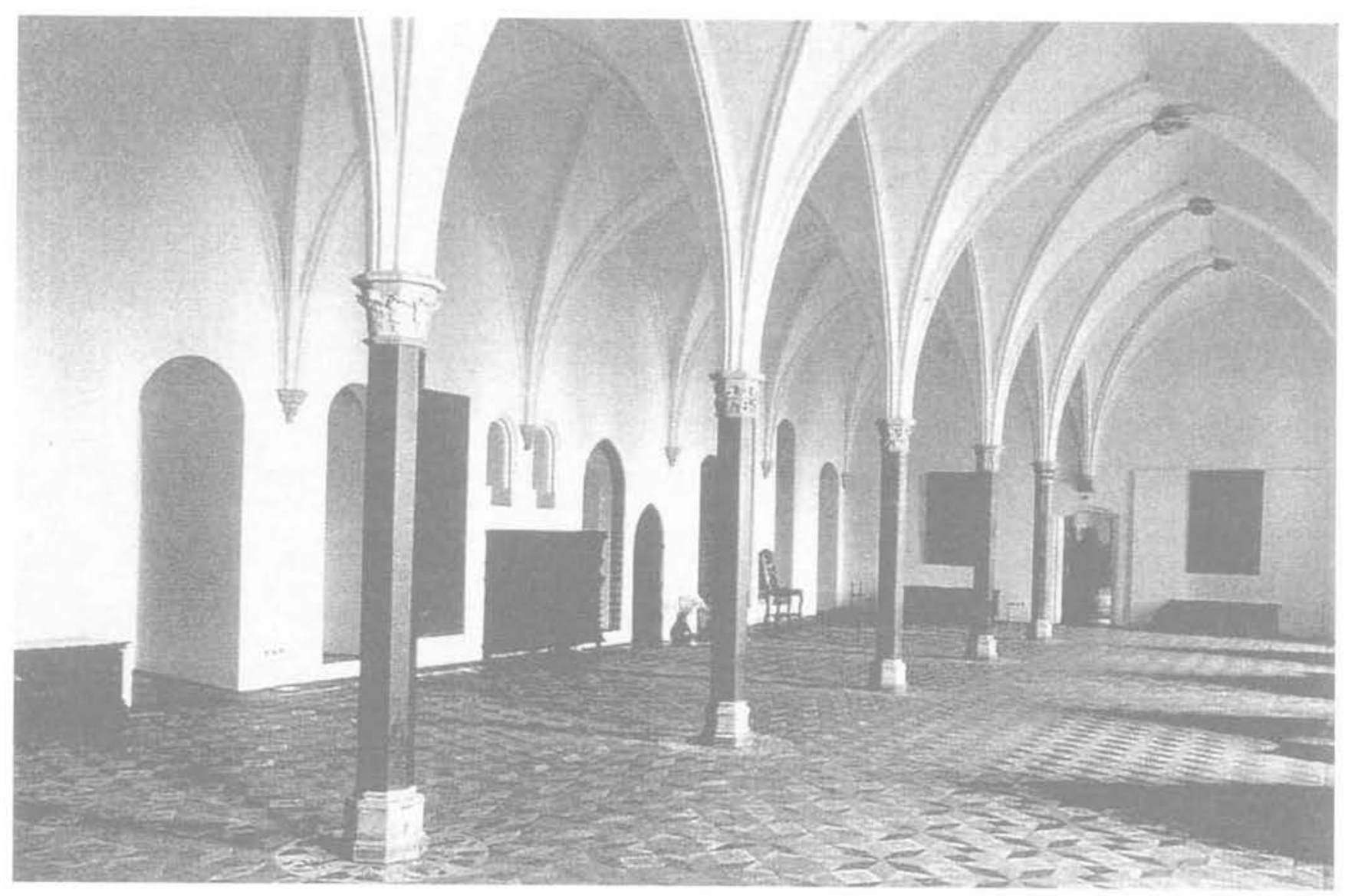

riG. 9 Le Grand Réfectoire (photo R. Czewinski et A. Stachurski). The Great Refectory.

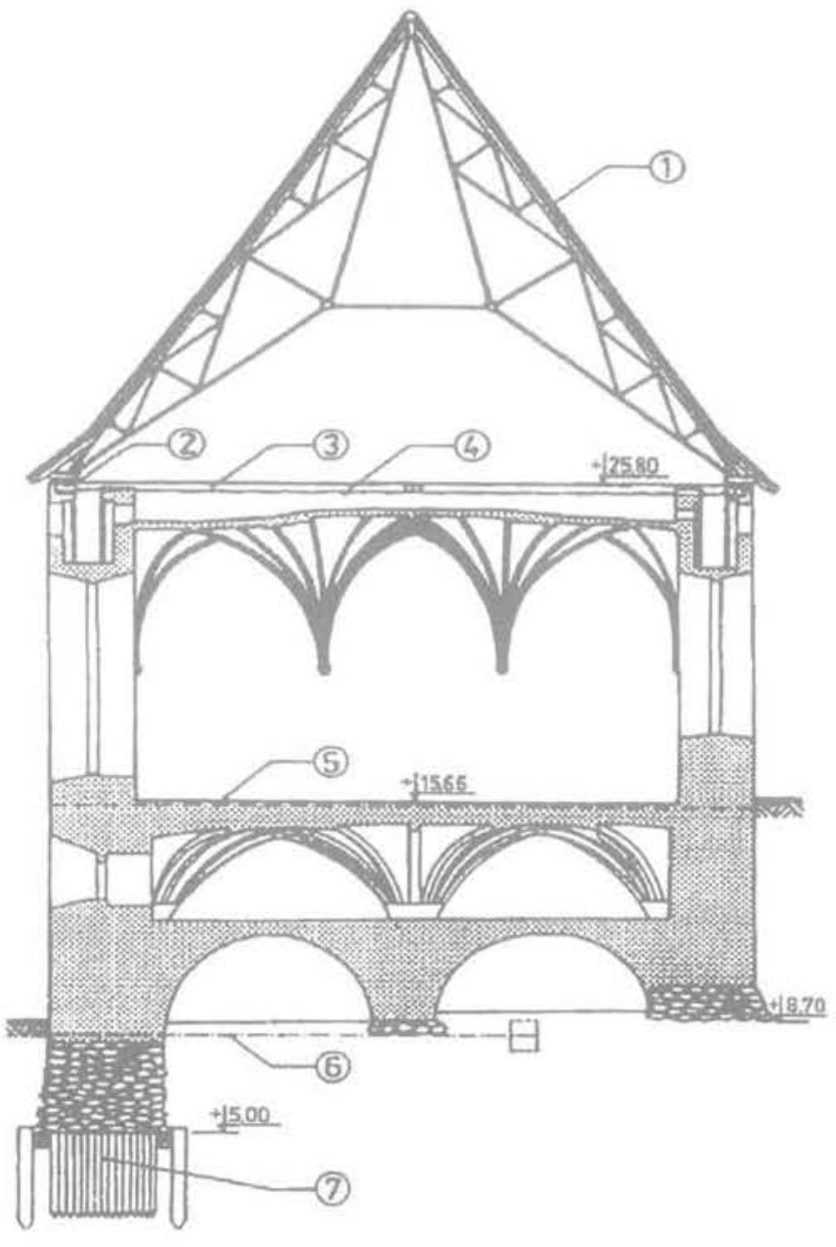

FG.10 Section transversale de l'aile Ouest avec toit actuel.

Cross section of the West wing with actual roof.

1. Couverture ardoises sur charpente acier.

tile skin on steel framework.

2. Cadre-porteur en béton armé.

reinforced concrete bearing frame

3. Grille en béton armé au-dessus de la voüte du réfectoire. concrete grid located above the arches of the mess hall.

4. Entraits en acier, ancrés sur les cadres porteurs,

steel tie backs anchored to the concrete frame

5. Entraits en acier, sous le plancher du réfectoire.

steel tie backs anchored under the floor of the mess hall.

6. Entraits en acier, sous le plancher de caves,

steel tie backs under the floors of the cellar.

7. Pieux en bois sous le mur Ouest.

wooden piles under western wall. 
Elles apparurent nombreuses vers 1860, et il fallut entreprendre une opération de sauvegarde plus importante. Elle consista en la mise en œuvre d'un contrefort en pierre au sous-sol et des entraits au grenier (qui furent par la suite démontés vers 1900).

La régulation de la Vistule vers 1903 a aggravé la situation à la suite des effets hydrotechniques suivants : raccourcissement de la longueur du fleuve, augmentation de la pente et abaissement du niveau de l'eau dans les différentes branches du delta.

Les premiers signes de détérioration grave dans les murs et les voutes de la salle du Grand Réfectoire sont apparus en 1963. Le processus du sinistre a probablement commencé immédiatement après l'incendie de 1959 qui a eu lieu dans les ailes Ouest et Nord (cf. Fig. 10). A partir de cette époque, fissures, déplacements verticaux et horizontaux allaient en grandissant (cf. Fig. 14). Ces désordres étaient provoqués par l'écartement des murs et la rupture de voûtes historiques et constituaient une sérieuse menace d'effondrement. Il est alors devenu nécessaire d'entreprendre d'urgence des travaux de confortement, ainsi que de prendre enfin une décision pour la sauvegarde définitive du monument.

\section{Coupe géologique de la terrasse du Nogat}

Les forages exécutés ces dernières années, complétés par deux puits blindés de reconnaissance dans le voisinage immédiat du mur Ouest, ont permis de préciser la coupe du terrain au droit des fondations.

La figure 11 illustre les différences en niveau ẹt en nature de terrain du sol d'assise des fondations du mur Ouest (du côté du Nogat), et du mur Est (du côté de la cour de l'aile Ouest du Château Moyen. La différence de niveau des terrains contigus atteint $6,50 \mathrm{~m}$, celle des niveaux de la base des fondations $3,5 \mathrm{~m}$ environ. Le mur Est s'appuie sur une semelle, au-dessous de laquelle il y a une couche d'argile de $2 \mathrm{~m}$, ensuite on trouve le sable fin. La coupe géologique montre nettement une disposition en pente vers l'est du système géologique naturel. Au voisinage de la fondation du mur Ouest, le sol est formé de couches de tourbe, de sable avec éléments organiques et aussi d'une couche contenant un mélange de plusieurs sortes de sol, de matériaux de construction et de déchets. De fortes venues d'eau souterraine, malgré les injections et le pompage largement pratiqués, n'ont pas rendu possible l'exécution des petits puits à une profondeur inférieure au niveau de la fondation en pierre. Pour reconnaître le sol sous-jacent on a dû exécuter un forage.

La fondation du mur est établie sur un sol renforcé à l'aide de pieux en bois; les figures 10 et 11 montrent la situation de la fondation du mur Est. Son assise repose sur la couche de sable et correspond à peu près au niveau de l'eau souterraine.

\section{4}

\section{Fondation du mur Ouest du Grand Réfectoire}

Les examens effectués grâce aux deux puits exécutés en 1990 ont permis de connaître des éléments de base constituant la fondation du mur Ouest et de

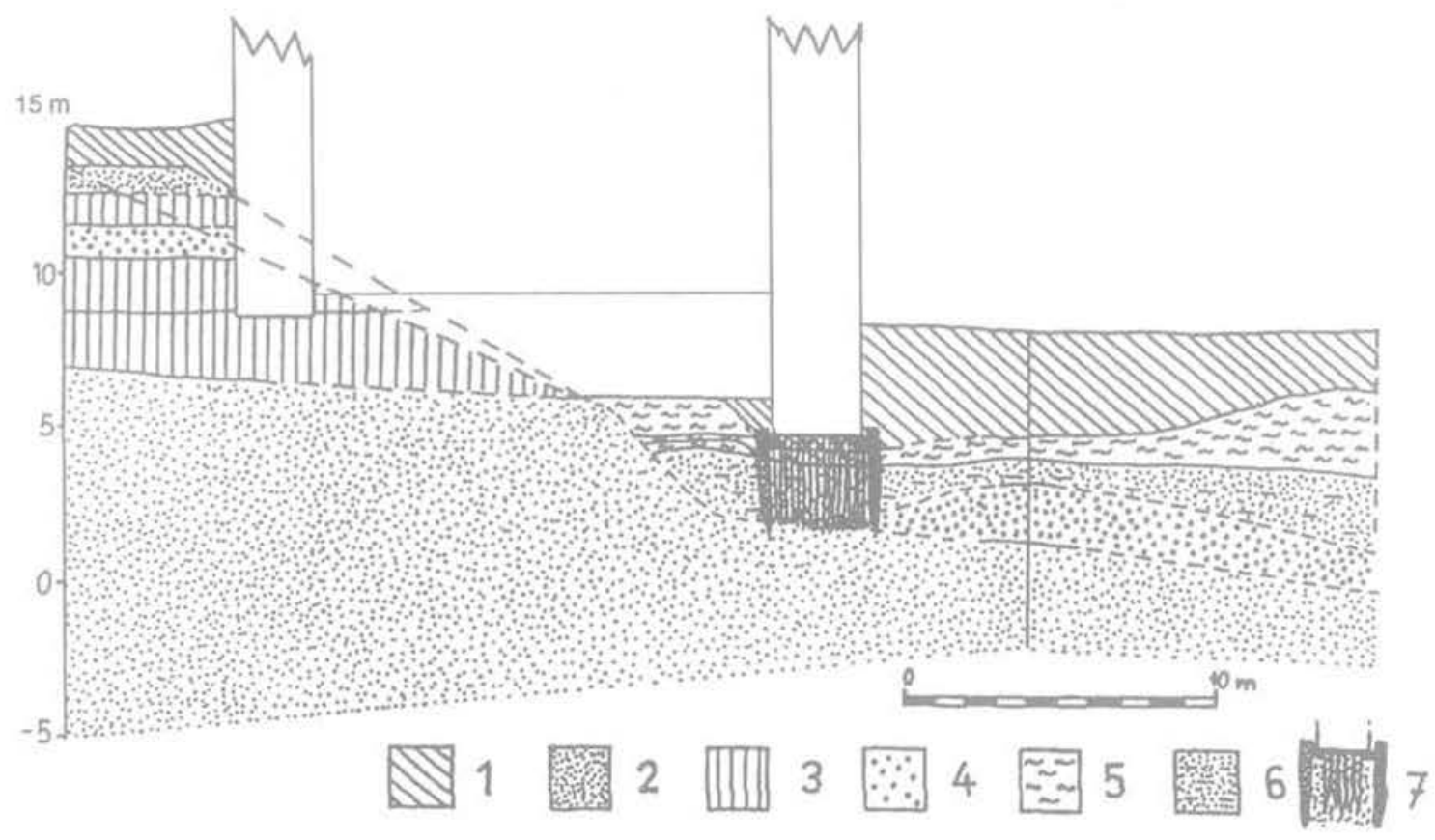

FG. 11 Coupe géologique de l'aile Ouest du Château Moyen.

Geological cross section under the western wing of the Middle Castle,

Cỏté droit -terrasse de Nogat; côté gauche - moraines, Right side - Nogat river terrace; left side - moraine.

1. Remblai, fill. 2. Sable fin, fine sand. 3. Argile, clay. 4. Gravier, gravel. 5. Vase, mud. 6. Sable moyen avec déchets bois, graded sand

with wood fragments. 7. Pieux en bois, wood piles. 
reconstituer le système porteur ancien. L'exécution des puits fut très difficile, le niveau de la nappe se trouvait au-dessus du niveau d'assise de la fondation. De plus, l'équilibre du mur fissuré et décollé du reste du château était menacé par ces travaux.

Pendant les travaux de reconnaissance, on a trouvé des pieux en bois dont l'existence était inconnue. La figure 12 présente la base du mur telle qu'elle peut être reconstituée.

Le mur en briques, de $3,10 \mathrm{~m}$ à $3,34 \mathrm{~m}$ d'épaisseur moyenne repose sur une fondation dont la partie haute est constituée de pierres liẻes à l'aide d'un mortier de chaux. Dans la partie basse l'espace entre les pierres est rempli d'argile.

La construction en bois, sur laquelle est posé le massif de fondation en pierres, commence à une cote de $5,45 \mathrm{~m}$ au-dessus du niveau de la mer.

Cette partie en bois se compose de deux parties qui ont été découvertes successivement.

A une distance de 30 à $65 \mathrm{~cm}$ à l'ouest du nu du mur on a découvert des pjeux en bois de $30 \mathrm{~cm}$ de diamètre chacun, espacés de $45 \mathrm{~cm}$. Derrière ceux-ci se trouvent des poutres horizontales, également en bois, de $50 \times 50 \mathrm{~cm}$ de section. Ces pieux et ces poutres sont en chêne. Ce dispositif de forme rectangulaire constitue une enceinte analogue à un batardeau à claire-yoie.

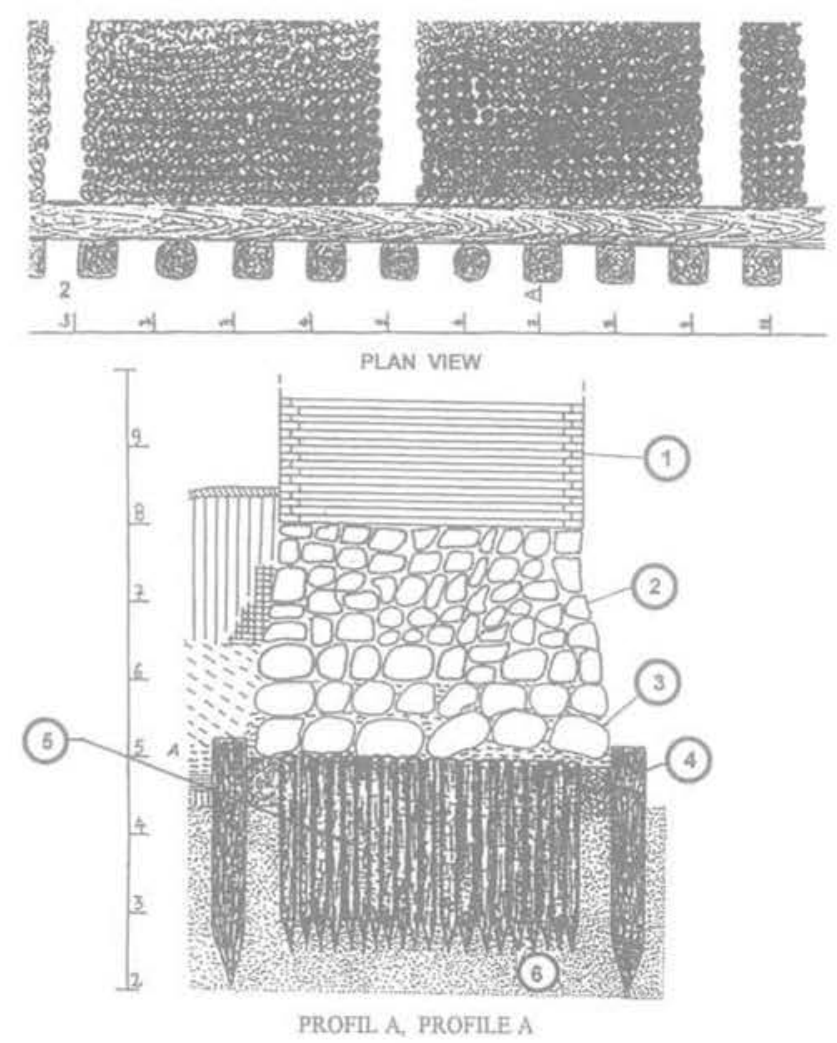

FG. 12 Fondation du mur Ouest du Grand Réfectoire - reconstitution.

Foundation of the western wall of the Great Mess Hall.

1. Mur en briques, brick wall. 2. Cailloux liés au mortier de chaux, stones in lie mortar. 3. Cailloux avec argile, stones and clay. 4. Pieux en bois de chêne $\varnothing 30 \mathrm{~cm}$, oak piles $\emptyset 30 \mathrm{~cm}$. 5 . Pieux en bois résineux $\varnothing 18$ à $22 \mathrm{~cm}$, resinous wood piles. 6 . Sable moyen, medium dense sand.
A l'intérieur de cette enceinte, directement sous la fondation en pierres avec liant d'argile on a découvert des pieux en bois résineux enfoncés à très petite distance les uns des autres dans un sol sableux.

Ces «micropieux $n$ ont un diamètre de 18 à $22 \mathrm{~cm}$.

La file extérieure était tangente à la poutre horizontale qui les protégeait d'un éventuel déversement vers l'extérieur.

On avait ainsi constitué sous la maçonnerie de pierre une forêt de amicropieux $\nu$ pratiquement jointifs, entourés par l'enceinte des pieux de $30 \mathrm{~cm}$.

Pendant les travaux, on a découvert que cette «forêt de micropieux $\#$ comportait des intervalles de 40 à $50 \mathrm{~cm}$ remplis de sable, régulièrement répartis dans le sens perpendiculaire au fleuve. Un tel système permet de laisser libre cours à l'eau souterraine venant de la cour et du mur Est de l'aile Ouest du Château Moyen et se dirigeant vers le Nogat.

Étant donné les difficultés d'investigations et les risques de détériorations de la construction, on n'a pu examiner le longueur des pieux (ayant peut-être $3 \mathrm{~m}$ ).

Nous ne savons pas si les constructeurs de l'époque ont commencé par la mise en place des «micropieux n en bois ou celle de l'enceinte périphérique. Cependant, compte tenu de l'existence des tranchées drainantes de sable, il parait logique d'admettre qu'ils ont commencé par l'enceinte périphérique, permettant de mieux contrôler la mise en place des micropieux bois et des drains sableux.

Par ailleurs, la présence de cette enceinte a dû augmenter l'effet de compactage dû à la mise en place des " micropieux» bois tout en empèchant le déversement de ces éléments de faible diamètre.

En effet, la construction donne l'impression qu'on a fait un grand effort pour améliorer le substratum humide et lâche qui existait, par le battage de pieux qui ont incontestablement densifié le terrain.

Vues sous l'angle des techniques actuelles, ces dispositions suscitent un grand respect pour les constructeurs du château.

L'état des éléments examinés varie. Les pieux et les poutres horizontales en chêne sont en bon état, à l'exception d'unè très faible dégradation en surface. Leur structure reste solide et dense, leur couleur est noire. Cette coloration est classique pour le chêne ayant séjourné très longtemps sous l'eau. C'est ce que l'on appelle en Pologne le chéne unoir ». Les spécialistes n'ont aucun reproche à formuler au sujet de ce type de construction.

La situation est tout à fait différente quand il s'agit des micropieux en bois résineux. Une importante partie retrouvée dans le puits $n^{\circ} 1$ (Fig. 13) était presque entièrement pourrie et sa structure, très poreuse, semblable à une éponge humide. II faut en chercher les causes dans le changement périodique de niveau d'eau souterraine qui faisait émerger le bois de moins bonne qualité que le chène, ou bien dans la présence de bactéries anaérobies qui l'avaient attaqué. Ainsi l'efficacité de ces micropieux en bois était devenue douteuse.

Par contre, l'état des pieux dans le puits $n^{\circ} 2$ était meilleur (Fig. 13), même les " micropieux 》 en bois résineux ont été trouvés en bon état. 


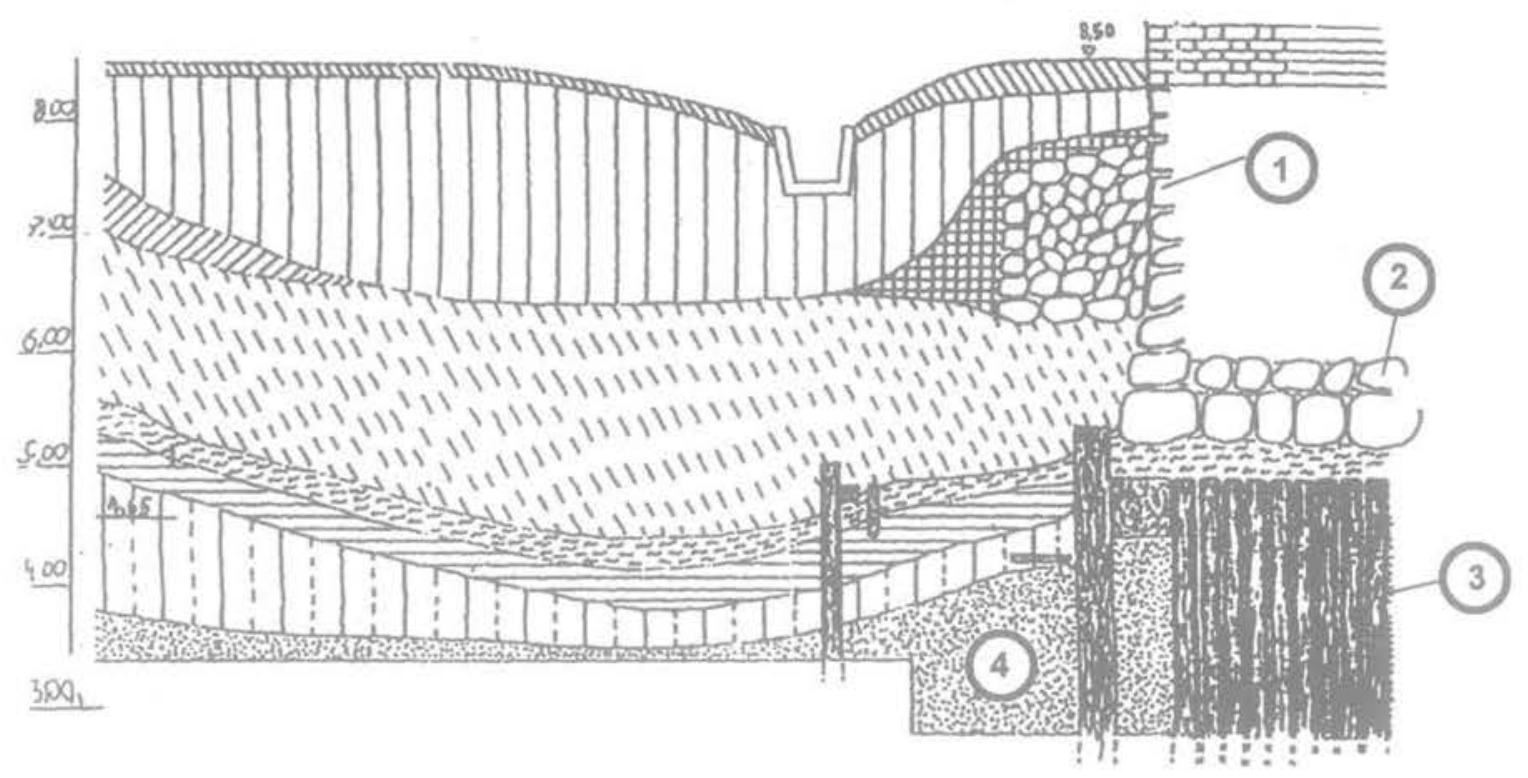

Humus contemporain, top soil.

T1 Humus avec gravats (après incendie 1959).

top soil with rubble (after the 1959 fire).

Humus très sableux avec gravats (moitié du XIXe siècle), very sandy top soil with rubble (middle 19th century).

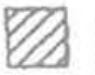

Humus avec gravats (moitié du XIXé siècle),

top soil with rubble (middle 19th century).

[1] Sable, argile, humus, gravats (XIVe:XIXe siècle),

=-2 Limon avec gravats (XIII-XIXe siècle),

silt with rubble (13th-19th century).

Limon gris-noir, naturel,

grey-black silt, original soil.

i] Limon gris sableux, sandy grey silt

Sable moyen, medium dense sand.

nQ: 13 Fondation du mur Ouest de l'aile Ouest du Château Moyen.

Foundation of the western wing, Middle Castle.

1. Cailloux liés au mortier de chaux, stones in lie mortar. 2. Cailloux avec argile, stones with clay.

3. Pieux en bois, wood piles, 4. Sable moyen, medium dense sand.

\section{5}

\section{Causes des désordres observés dans l'ouvrage}

La dégradation des voûtes, des linteaux et des murs dans l'aile Ouest résulte des déplacements excessifs des fondations. La forme, l'allure et la grandeur des fentes et des fissures montrent bien que ces désordres sont la conséquence de tassements différentiels et de déplacement verticaux et horizontaux.

Les mouvements des fondations qui ont engendré ces désordres en mettant le bâtiment entier en danger sont imputables aux modifications des caractéristiques du sol sous les fondations.
Les désordres observés au cours des siècles dans les murs, qui ont été renforcés à de nombreuses reprises, résultent de la trop faible résistance des couches superficielles du terrain eu égard aux charges transmises.

L'abaissement sensible des niveaux de l'eau dans le fleuve Nogat, après sa régulation et le raccourcissement du cours de la Vistule au début du XXe siècle, provoqua le rabattement de la nappe phréatique dans la zone du mur Ouest du château.

Cette modification du niveau d'eau provoqua tassement et fluage des couches en direction du fleuve, en raison de leur pendage.

Les fondations en bois qui se trouvaient à l'origine complètement sous l'eau ont émergé partiellement, et il en est résulté la destruction biologique des pieux et des poutres de liaison en bois tendre. 
L'augmentation récente des tassements et des fissures dans les murs et les voûtes pouvaient conduire à une perte de stabilité et à la ruine totale de l'aile Ouest.

Les travaux confortatifs, pour être efficaces, devaient donc en priorité concerner les fondations. Les autres réfections concernant les murs et les voûtes pouvant être réalisées plus tard, une fois la stabilisation des fondations obtenue.

\section{6}

\section{Propositions de renforcement du mur Ouest du château}

Les essais faits dans le passé, les propositions présentées et les renforcements réalisés alors ont toujours eu un caractère d'urgence, sans faire disparaître les causes. Ils se limitajent à supprimer les effets et à protéger la construction contre les conséquences du déplacement des fondations sans améliorer réellement ces dernières. Ils consistaient à réaliser des contreforts en pierres, (vers le milieu du XIX" siècle), à mettre en place des tirants à des niveaux différents et des ancrages intérieurs dans les fondations, à exécuter des renforcements des murs et, enfin, à mettre en œuvre des clefs dans les maçonneries, ainsi que des étançons et des bracons. On avait installé également un système complet d'observations et de mesures de déformations.

Les essais d'injection des sols par silicate tentés en 1972 n'ont pas pu être continués pour des raisons techniques.

Il y a quelques années, on a constaté de très graves dommages et l'accélération des tassements (cf. Fig. 14 et 15), ce qui a enfin obligé à étudier des méthodes de renforcement plus efficaces. On a procédé alors à de nombreuses analyses de stabilité et de résistance de toute la construction, on a envisagé plusieurs variantes possibles de confortement des fondations.

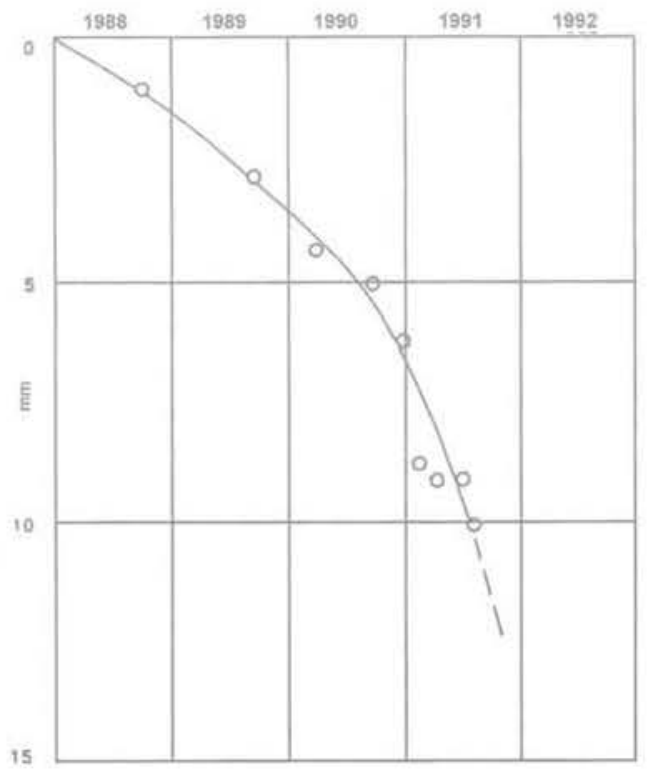

FG. 14 Tassement du mur Ouest depuis 1988. Settlement of the western wall since 1988.
Certains avaient proposé l'utilisation de pieux de type Mega, d'autres des injections telles que le jet grouting. On avait envisagé également la congélation du sol avec reprise en sous-œuvre traditionnelle.

Finalement, on a adopté une solution par micropieux (cf. Fig. 16 et 17) qui a été réalisée en 1992.

\section{7}

\section{Analyse de la stabilité de la fondation et de l'aile Ouest}

La réparation et le renforcement des murs du Grand Réfectoire, faits à plusieurs reprises, consistaient à reconstruire des murs, à installer des contreforts et des entraits, à ancrer et aussi à mettre en place des cadres porteurs, ainsi qu'à changer la structure du toit, ce qui a pu entrainer quelques modifications dans le comportement du gros ceuvre. Dans une certaine mesure, ces travaux ont pu améliorer la stabilité de la construction et limiter les déformations dangereuses. Par contre, ils n'ont pas pu supprimer la cause principale des dégradations et, notamment, celle qui était liée à l'affaissement du sol; ils n'ont pas eu, non plus, d'influence sur la modification de la charge des fondations.

Une analyse détaillée de la construction, prenant en considération diverses variantes possibles en ce qui concerne les charges et l'interaction des éléments de renforcements a confirmé que les déplacements futurs des fondations pouvaient constituer une menace pour le bâtiment. Le tassement du mur Ouest du Grand Réfectoire, mesuré dans les années 1988-1991 (Fig. 14) était de 2 à $4 \mathrm{~mm}$ par an, avec une nette tendance à augmenter. Alors que, si les fondations sont stables, les murs (sauf les éléments directement en contact avec la charpente) remplissent largement les exigences de résistance et de stabilité.

L'analyse des charges importantes à transmettre montre que les massifs de fondations ètaient insuffisants et devaient être renforcés.

Selon les différents cas, les efforts à la base des fondations en maçonnerie de pierre ont pour valeur:

- charge horizontale $\mathrm{H}=50 \mathrm{kN} / \mathrm{ml}$;

- charge verticale $V=1008$ à $1300 \mathrm{kN} / \mathrm{ml}$;

- moment de flexion $\mathrm{M}=279$ à $820 \mathrm{kN} \cdot \mathrm{m} / \mathrm{ml}$.

En tête de la fondation en pierres hourdées à la chaux, ayant une largeur de $3,10 \mathrm{~m}$ la contrainte de compression est de $916 \mathrm{kN} / \mathrm{m}^{2}(0,916 \mathrm{MPa})$ ce qui est très élevé.

Par ailleurs, la zone en traction s'étend sur $61 \mathrm{~cm}$, ce qui est préoccupant lorsque le liant entre pierres présente une faible résistance.

Pour une largeur totale de la base $B=4,10 \mathrm{~m}$, la pression sur le sol varie entre les limites suivantes:

$$
24,2 \mathrm{kN} / \mathrm{m}^{2}<\sigma<610 \mathrm{kN} / \mathrm{m}^{2}
$$

Pour une largeur totale $\mathrm{B}^{\prime}=3,80 \mathrm{~m}$

$$
1,3 \mathrm{kN} / \mathrm{m}^{2}<\sigma<775 \mathrm{kN} / \mathrm{m}^{2}
$$

Ces contraintes sont également très élevées pour un sable, même compacté par les pieux en bois, et totalement inadmissibles, si les pieux en bois sont pourris.

La protection et le renforcement des voûtes et de certains des éléments des murs transversaux constituent un problème secondaire, à résoudre naturellement après avoir stabilisé la fondation du mur Ouest. 


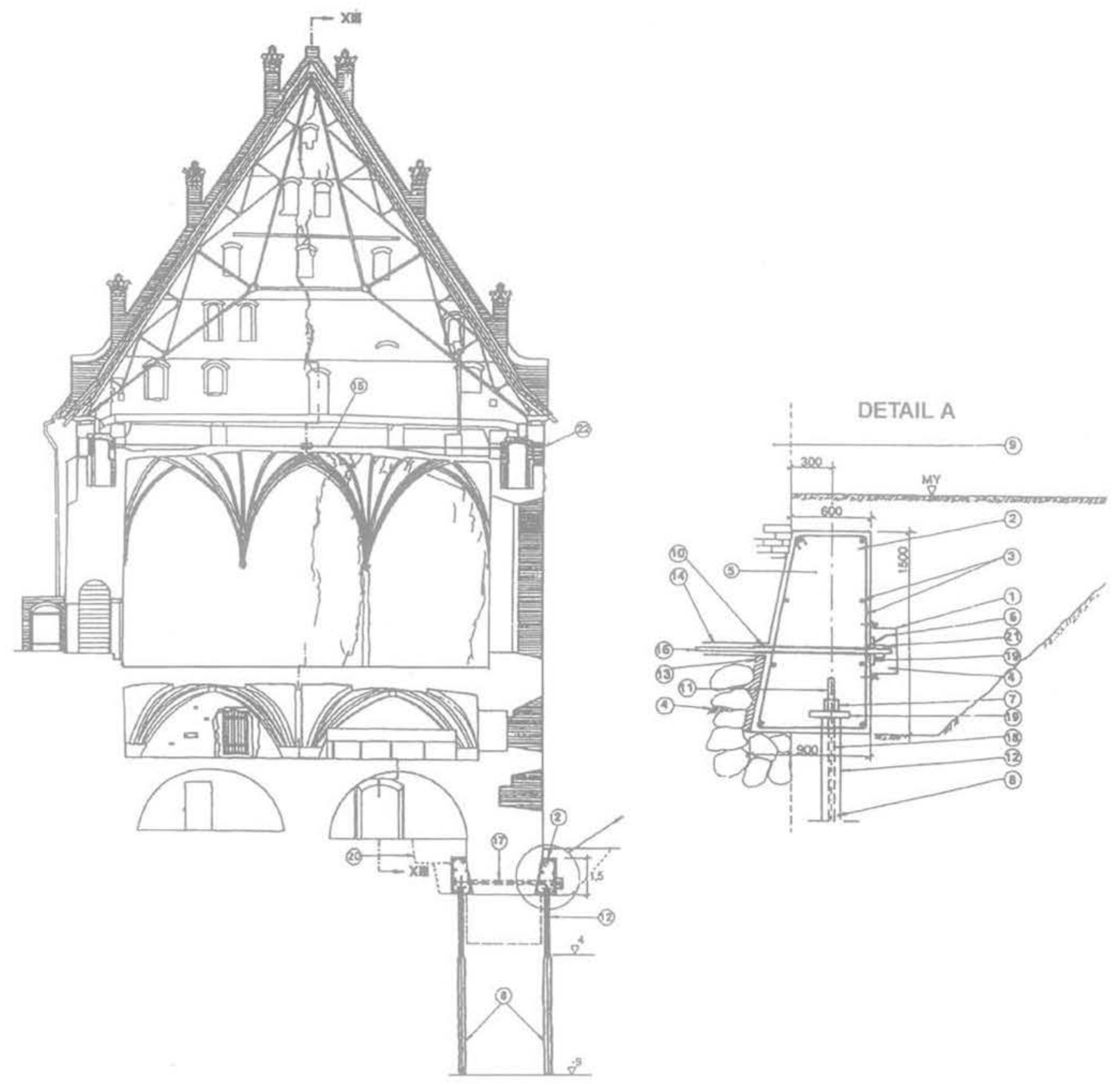

FIG. 15 Section transversale: fissures bien visibles, proposition de renforcement. Cross section : visible cracks, proposal for reinforcement.

1. Boite-élérnent de protection contre la corrosion, box-corrosion protection element.

2. Sablière supérieure en béton armé.

upper reinforced concrete tie beam.

3. Armature, reinforcement.

4. Mortier ciment, en cas de nécessité, eventual cement paste.

5. Béton K 30 , concrete $\mathrm{K} 30$.

6. Tube d'injection, injection tube.

7. Ecrou, screw.

8. Pieu TNA tous les mètres, TNA pile every meter,

9. Mur en briques, brick wall.

10. Tuyau en plastique, plastic pipe.
11. Barre GWS Ø36, GWS Ø 36 bar.

12. Tuyau de mise en place $\varnothing 165$, guide pipe $\varnothing 165$.

13. Béton projeté, shotcrete.

14. Trou $\varnothing 80 \mathrm{~mm}, 80 \mathrm{~mm}$ hole.

15. Cable de compression type GWS, post tension cable.

16. Barre GWS Ø 36, GWS $\varnothing 36$ bar.

17. Ancrage transversal, transverse anchor.

18. Mortier de ciment, cement paste.

19. Plaque en acier, steel plate.

20. Excavation locale, local excavation.

21. Boulon d'ancrage, tie rod nut.

22. Blocage du tirant, tie rod anchor. 


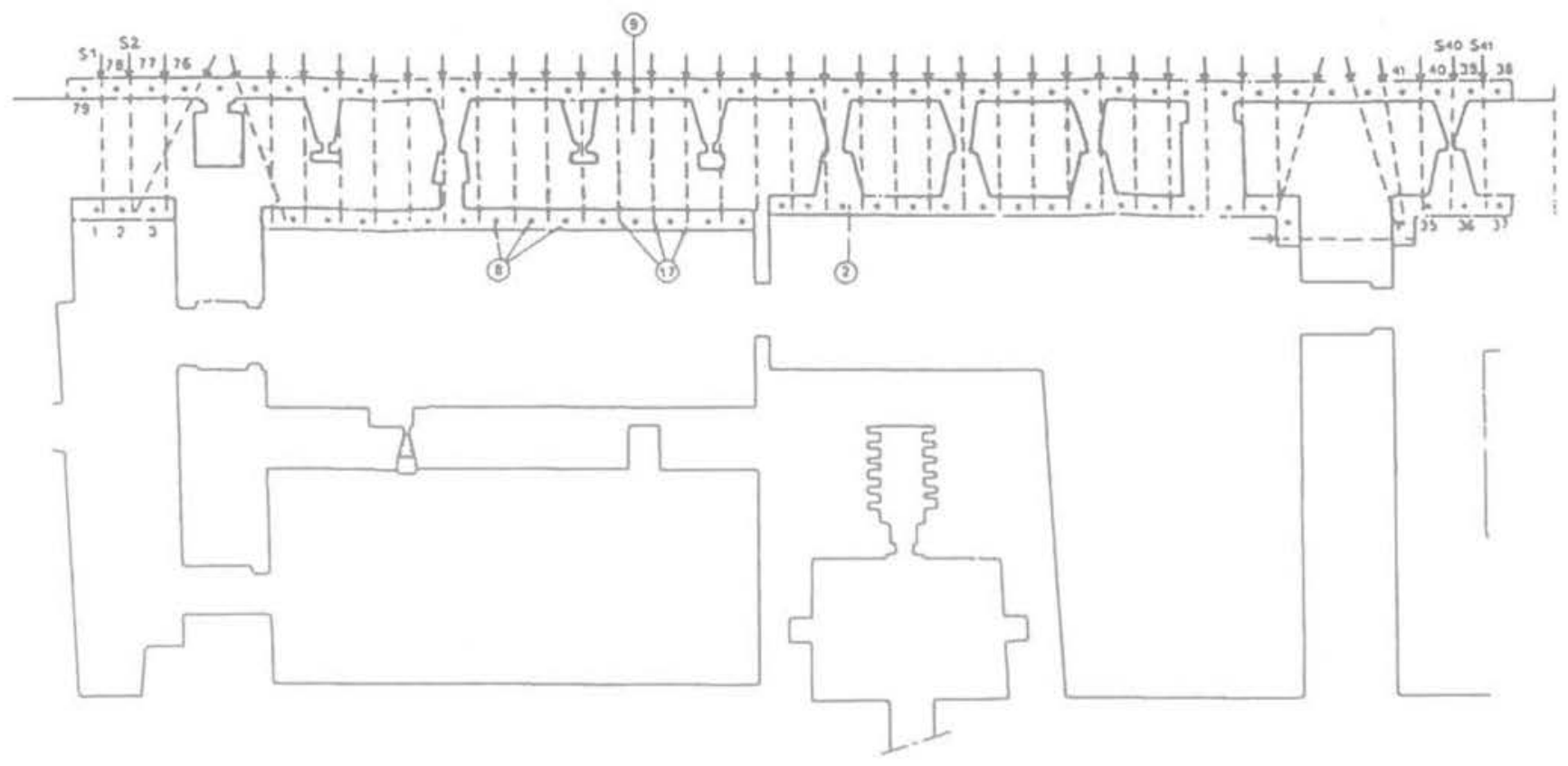

Flo. 16 Proposition de renforcement: plan. Reinforcing proposal: plan view.

\section{8}

\section{Conception du renforcement réalisé}

La dégradation permanente de la fondation existante, l'amplitude grandissante des déplacements verticaux et horizontaux, ainsi que la menace inévitable de catastrophe, ont conduit à adopter d'urgence une méthode de confortement efficace.

L'exigence primordiale était d'assurer la possibilité de transmettre la charge de la fondation actuelle au système de renforcement choisi.

Les solutions pouvant être retenues pour cela devraient remplir en plus quelques exigences constructives, telles que:

- adaptation à des conditions existantes de géométrie, de stabilité, de rupture, ainsi qu'à celles du sol et de l'eau, etc.;

- possibilité technologique d'assurer les conditions prévues dans le projet;

- tenue garantie du renforcement (stabilité et résistance à des déplacements) ;

- garantie de déplacements minimaux pendant les travaux.

Les difficultés principales qui ont été rencontrées dans le cas du mur Ouest du Château Moyen ont été les suivantes:

- grande épaisseur du mur $(3,10 \mathrm{~m})$ et charge assez importante $(\mathrm{V}=1300 \mathrm{kN} / \mathrm{ml}, \mathrm{M}=820 \mathrm{kN} . \mathrm{m} / \mathrm{ml})$;

- fondation en pierres liées avec un mortier de faible résistance (dans la partie haute mortier de chaux, dans la partie basse argile);

- pieux en bois à différents degré de décomposition (cf. constats dans les puits $n^{\circ} 1$ et $n^{\circ} 2$ );

- accès difficile pour les machines en raison de voûtes très basses dans les caves;
- existence d'une couche de sol à faible portance, directement au-dessous de la base de la fondation;

- difficulté d'estimer la résistance réelle du mur (possibilité de points faibles dans le mur et probabilité d'une faible résistance du mortier);

- possibilité d'existence d'obstacles à côté de la fondation, tels que blocs de pierre, grumes et anciens contreforts ;

- état alarmant des voûtes et graves fissures dans les murs transversaux;

- sensibilité de la construction à des chocs et aux changements de niveaux de la nappe phréatique;

- impossibilité de réaliser des fouilles à ciel ouvert pour reprise en sous-cuvre traditionnelle;

- épaisseur du terrain de recouvrement très faible au-dessus du niveau d'exécution des travaux, donc impossibilité d'injection à haute pression;

- contrôle difficile des éléments exécutés sous l'eau;

- ignorance des causes des déplacements horizontaux observés probablement déjà au siècle dernier. Étaient-ils dus à la pente naturelle entre le mur Est, le mur Ouest et le Nogat? à l'inclinaison des couches? à l'écoulement de l'eau? à l'action d'étrésillonnement des charges transmises à partir des voûtes et des arcs en raison du mauvais état des entraits et du manque de contreforts d'origine?

Les remarques et les objections présentées ci-dessus ont empêché le recours à la plupart des méthodes théoriquement possible comme, par exemple, celles qui exigeaient un perçage de la fondation ou qui auraient pu provoquer un ébranlement ou un rabattement du niveau de l'eau. On a dû également éliminer les injections nécessitant des machines dont l'accès aux caves aurait été difficile.

Finalement, on a décidé de mettre en œuvre une méthode proposée par l'entreprise suédoise STABILATOR. 


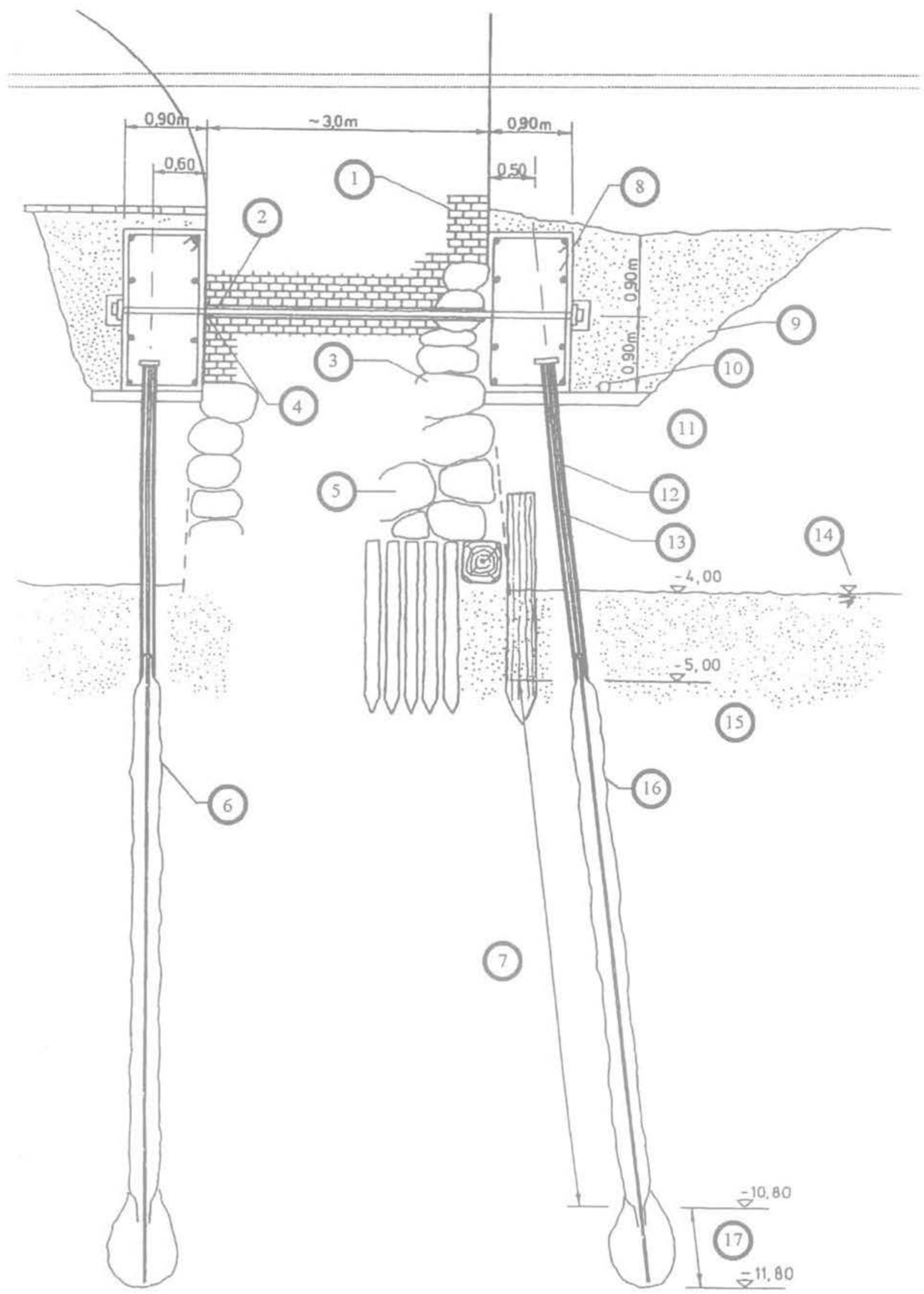

FlG.17 Renforcement du mur Ouest du Château Moyen par des pieux-profil caractéristique. Reinforcing of the western wall of the Middle Castle with piles - typical profile.

1. Mur en briques, brick wall. 2. Cables de compression type VSL dans un tuyau en plastique, prestressing lines type VSL in plastic pipe. 3. Mur en cailloux de chant, rubble wall. 4. Trou du forage, coredrill hole $\varnothing 85 \mathrm{~mm}$. 5. Pieux en bois - fragment médiéval, wooden grillwork, wooden piles (stakes) - the medieval foundation. 6. Micropieu vertical type TNA $30 \mathrm{~cm}$ de diamètre vertical, micropile type TNA. diam. $30 \mathrm{~cm}$ vertical. 7 . Zone active de pieu, the zone of bearing pile mantle. 8. Poutre sablière béton $\mathrm{K} 30$, the girt beam concrete $K 30$.
9. Remblai latéral, backfill. 10. Tube de drainage, drain pipe. 11. Remblai de sable et d'argile (couche ancienne), brick debris Joam backfill (historical layer). 12. Tube de forage $168 \mathrm{~mm}$ de diamètre, drilling tube $0168 \mathrm{~mm}$. 13. Tube de guidage type GWS, guide pipe type GWS $\emptyset 36 \mathrm{~mm}$. 14. Nappe phréatique, groundwater level. 15. Sable fin, fine sand. 16. Micropieu type TNA c $85 \mathrm{~mm} 40 \mathrm{~cm}$ de diamètre, inclinaison 10:1, microphile type TNA c $85 \mathrm{~mm}$, diam. $40 \mathrm{~cm}$, rake 10:1.17. Zone de la base de pieu, the zone of pile tip. 
Les éléments essentiels de cette solution sont présentés sur les figures 16 et 17.

Cette méthode consiste à implanter des micropieux de deux côtés du mur et à les relier ensuite à l'aide de grandes poutres sablières en béton armé dont la liaison avec le mur de la fondation est assurée par des câbles de compression transversaux.

Les micropieux ont dû être enfoncés le plus près possible du nu du mur, pratiquement à une distance de $0,6 \mathrm{~m}$ à $0,8 \mathrm{~m}$ exceptionnellement à $1,2 \mathrm{~m}$. Ils ont été descendus à une profondeur minimale de $12 \mathrm{~m}$.

Les micropieux sont fichés dans les couches de sable profondes. Les fouilles près des murs ont été exécutées parties par parties jusqu'à une profondeur de $2 \mathrm{~m}$ au-dessous du niveau du terrain. Les ouvertures dans les murs, faites en vue d'y passer les câbles de compression en acier, ont été exécutées par une méthode dé forage plein trou sans choc.

Les câbles ont été tirés par les ouvertures et ont été ensuite injectés. De deux côtés du mur, on a réalisé des poutres en béton armé très massives. Après le durcissement du béton on a mis en tension les câbles, comprimant ainsi les poutres sur le mur existant et en créant une construction monolithique. La mise en œuyre de ce système rend possible la transmission de charges des voûtes du toit et des murs sur les micropieux.

Ceux-ci ont un diamètre de $30 \mathrm{~cm}$. Leur extrémité inférieure est à environ $6 \mathrm{~m}$ au-dessous de la base des maçonneries de pierres. Pendant les essais, les micropieux ont montré une capacité portante de 840 et $890 \mathrm{kN}$. Le tassement sous la charge d'utilisation n'a pas dépassé $5 \mathrm{~mm}$.

Pendant l'exécution des pieux, les travaux de forage ont été menés à l'aide de tubes de 140 et de $169 \mathrm{~mm}$ de diamètre, avec une quantité minimale de boue de forage. Bien que les travaux aient été conduits d'une manière exemplaire, on n'a pas pu éviter quelques tassements supplémentaires qui ont atteint environ $15 \mathrm{~mm}$ en moyenne. Le sable fin à granularité uniforme, que I'on devait traverser, s'est révélé sensible au phénomène de liquéfaction.

Les doutes antérieurs concernant l'utilisation de la méthode d'injection (jet grouting ou autres), jugée dangereuse pour la construction, se sont révélés parfaitement justifiés, car l'édifice n'aurait probablement pas supporté une telle méthode de confortement.
Les travaux ont été exécutés au cours du deuxième semestre de 1992. Cette réalisation a été possible avant tout grâce aux machines spéciales de forage (appelées " souris de cave ") adaptées au travail dans des locaux très bas, et aussi, grâce à des équipements spéciaux. ainsi qu'à l'expérience de l'entreprise qui avait déjà réalisé des travaux analogues en Suède.

Les craintes qui pouvaient résulter de l'utilisation de cette méthode concernaient deux problèmes: la qualité du mur de fondation et la résistance des micropieux vis-à-vis d'une éventuelle charge horizontale pouvant être provoquée par le fluage de terrain.

Le premier est lié à la transmission de la charge verticale par des forces tangentes entre le mur et les poutres sablières supérieures, aux éventuelles fissures et à son fluage qui aurait pu induire une perte des forces de précontrainte. Heureusement, la qualité du mur pendant le forage plein trou (câblage de compression) s'est révélée très bonne, en suscitant une admiration pour les constructeurs d'antan.

Le mur possédait des encoches qui ont éliminé la nécessité de faire des entailles.

Il est difficile de savoir si la résistance des micropieux exécutés est suffisante pour faire face aux déplacements horizontaux éventuels. Quoi qu'il en soit, l'utilisation de sablières supérieures et leur jonction avec les pieux devrait améliorer nettement la situation antérieure.

L'effet immédiat de ce renforcement a été indiscutable et remarquable, son efficacité est prouvée par les mesures de déplacements. Une appréciation définitive du système adopté sera possible dans quelques années.

Le transfert des charges vers la nouvelle fondation ne s'opère pas immédiatement, mais s'effectue progressivement, au fur et à mesure des modifications des contraintes internes de la structure existante conservée par suite de l'évolution de la liaison constructions anciennes-travaux neufs.

Il s'agit d'un phénomène classique bien connu. Ce dernier peut s'aggraver en raison de modifications internes de la structure conservée. En effet, certaines parties de cette dernière peuvent se dégrader progressivement dans le temps et entraîner de nouveaux transferts et charges internes et, de ce fait, des modifications des charges transmises au confortement réalisé.

\section{Bibliographie}

Ajdukiewicz A., Klaszczewicz A., Hulimka I. - Analiza ustroju nosnego skrzydla zachodniego Zamku Sredniego w Malborku. Inzynieria $i$ Budownictwo n' 8/1992 s. 276-279

Albrecht J. - Problemy wzmacniania sciany zachodniej Zamku Sredniego w Malborku. Inzynieria i Budownictwo $n^{\circ} 7 / 1991$.

Documentation de l'entreprise STABIL.A. TOR Conc. - Le renforcement de la fon- dation du mur Ouest du Chấteau Moyen de Malbork.

Documentation scientifique et technique déposée dans les archives du Musée du Château de Malbork.

Najder T. - The works of strengthening of the western wing in Malbork Middle Castel. III Int. Seminar on Renovation and Improvements to Existing Quay Structures, Gdansk, 1993, pp. 57-63.
Pawlowski A.J. - Dotychczasowe wyniki badan geologicznych $i$ archeologicznoarchitektoniczych prowadzonych w rejonie sciany zachodniej skrzydla zachodniego Zamku Sredniego w Malborku. Prace Muzeum Zamkowego w Malborku, 1989.

Publikacje dotyczace historii i zabytkow Zamku Malborskiego. 